\title{
Tartamudez y Trabajo: Un Estudio Exploratorio
}

\author{
Stuttering and Work: An Exploratory Study
}

\author{
Margarita Hernández Rosado ${ }^{{ }^{*}}$, \& Miguel E. Martínez Lugo ${ }^{2}$ \\ 1 Carlos Albizu University, San Juan, Puerto Rico. (D) https://orcid.org/0000-0002-0611-5111 \\ 2 Carlos Albizu University, San Juan, Puerto Rico. (D) https://orcid.org/0000-0001-5586-2327 \\ * Correspondencia: mhernandez@albizu.edu
}

Recibido: 3 junio 2021 | Aceptado: 29 julio 2021 | Publicado: 13 agosto 2021

\section{WWW.REVISTACARIBENADEPSICOLOGIA.COM}

\section{Citar como:}

Hernández Rosado, M., \& Martínez Lugo, M. E. (2021). Tartamudez y trabajo: Un estudio exploratorio. Revista Caribeña de Psicología, 5, e5607. https://doi.org/10.37226/rcp.v5i1.5607

\section{RESUMEN}

Las organizaciones de hoy día buscan ser unas donde se valore la diversidad y se practique la inclusión. Muchas se enfocan en elementos como la cultura, el género, la edad, pero no prestan atención a otras fuentes de diversidad como puede ser la tartamudez. El objetivo de este estudio fue explorar las experiencias en el contexto de trabajo que experimenta un grupo de 13 personas con tartamudez. La muestra fue una por conveniencia y la participación consistió en contestar dos cuestionarios para obtener los datos pertinentes a nuestro objetivo investigativo. Los resultados nos indican que las personas con tartamudez que formaron parte del estudio confrontan problemas en la relación con sus compañeros de trabajo, en la realización de las tareas asociadas a su puesto y obstáculos para poder progresar en la organización. De igual modo, también indicaron que si no fuera por su tartamudez entienden que tendrían una experiencia de trabajo diferente. Estos resultados nos plantean el reto de ser más inclusivos en los contextos laborales y concienciar más a los empleados sobre la diversidad funcional.

Palabras Claves: diversidad, inclusión, tartamudez, trabajo

\begin{abstract}
Today organizations are trying to be more aware of human diversity and be more inclusive. Many organizations focus their attention on culture, gender, age, but they ignore other sources of diversity such as stuttering. This study aimed to explore the work experiences of 13 persons with stuttering. The sampling method was by convenience, and participants were asked to answer two questionnaires. The results showed that people with stuttering confront problems in their relations with coworkers, performing their job duties, and advancing in the organizations. Also, they inform that without stuttering, their work experience will be different. The results challenge being more inclusive in workplace settings and creating awareness of functional diversity issues.
\end{abstract}

Keywords: diversity, inclusion, stuttering, work

\section{INTRODUCCIÓN}

Los contextos laborales de hoy día prestan mucha atención a los diferentes elementos que componen la diversidad y las formas en cómo fomentar la inclusión de los diferentes grupos que componen la fuerza de trabajo. El desarrollo de la competencia del manejo de la diversidad, tanto a nivel individual como organizacional, se considera crítica e importante para poder mantener la competitividad en un 
mercado global y en un mercado laboral de empleados diversos (De Anca \& Vázquez, 2007). Jayne y Dipboye (2004) proponen que el cambio de la fuerza laboral a una más diversa debe conllevar esfuerzos organizacionales para contratar, retener y desarrollar empleados de grupos que están subrepresentados y crear estructuras para mantener programas de manejo de diversidad efectivos.

El estudio de la diversidad gira en torno a diversas perspectivas, que van desde unas muy amplias en las que se incluyen múltiples aspectos o elementos, hasta aproximaciones más limitadas de lo que es la diversidad. Esta variabilidad trae como resultado que no se encuentre un consenso sobre qué factores son los que debe tener su definición (Romero Flores \& Villanueva Flores, 2016). Una de las definiciones que más se cita en la literatura es la que presentó Triandis (2003) quien considera que la diversidad implica diferencias de etnias, razas, género, creencias religiosas, orientación sexual, discapacidad, edad, nación de origen y perspectivas culturales y personales. Por su parte, Ferdman (2014) indica que la diversidad es la representación de múltiples grupos en una organización o grupo de trabajo. De acuerdo con Romero Flores y Villanueva Flores (2016) valorar la diferencia implica ser conscientes de las diferencias humanas, comprenderlas y apreciarlas; lo cual produce que las personas se sientan valoradas y aceptadas como un recurso importante.

Otro tema que viene de la mano de la diversidad es la inclusión. La inclusión se relaciona con cuán bien la organización y sus miembros están completamente conectados y comprometidos y son utilizados tomando en consideración todas sus diferencias (Ferdman, 2014). La inclusión se hace posible a través de los comportamientos que se dan entre las personas que interactúan entre sí, las actitudes y los comportamientos de cada empleado y los valores, normas, prácticas y procesos de la organización (Ferdman, 2014). La inclusión conlleva crear contextos de trabajo donde las personas se sientan valoradas por sus diferencias y similitudes; es una manera de trabajar la diversidad y de beneficiarse de la misma (Ferdman, 2014).

Uno de los elementos que forma parte de la diversidad y las diferencias entre las personas es su capacidad para poderse comunicar. Las personas con tartamudez pertenecen a este grupo que presenta problemas para comunicarse en diferentes contextos, incluyendo su lugar de trabajo. Esta condición representa un desafío para la persona con tartamudez al desempeñar sus funciones como empleado y en sus relaciones con otras personas. Con este reto en mente, realizamos este estudio para conocer, de primera mano, cómo un grupo de personas con tartamudez vive su experiencia de trabajo. Específicamente nos interesó conocer qué dificultades encuentran, cómo es su relación con los compañeros de trabajo y cuál es su perspectiva de cómo su tartamudez incide en su rol como persona que trabaja.

\section{Tartamudez}

La comunicación es una de las capacidades más complejas del ser humano. Por medio de ella establecemos relaciones sociales, expresamos nuestros deseos, necesidades e inquietudes, y evidenciamos nuestras capacidades, preparación y nuestra cultura. Hay personas que carecen de una fluidez de habla adecuada para poder ejercer la función principal de la comunicación, que es poder expresarse con claridad. Estas personas presentan un trastorno de la fluidez conocido como tartamudez. La tartamudez es un desorden del habla que interrumpe la facilidad con la cual fluyen o discurren las palabras e interfiere con las interacciones verbales, así como también con la calidad de vida de las personas que la padecen. La American Speech, Hearing and Language Association (ASHA, 2021), define la tartamudez como un trastorno del habla, permanente o circunstancial, que se caracteriza por las repeticiones e interrupciones involuntarias en la emisión de palabras y puede ir acompañado de diversos movimientos del rostro y alteraciones respiratorias.

Las causas de la tartamudez se desconocen, sin embargo, se observa un alto nivel de ansiedad en estas personas. Según la Fundación Americana de la Tartamudez (2021), aproximadamente el $5 \%$ de los niños presenta episodios de falta de fluidez por seis meses o más. Tres de cada cuatro niños recuperan la fluidez antes de llegar a la adolescencia, quedando un $1 \%$ de esta población con un problema crónico. Las investigaciones apuntan a que la tartamudez se observa con más frecuencia en los varones (tres a cuatro veces más en varones que en hembras). 
Según el portal Mindyra.com (2021) la tartamudez afecta a aproximadamente el $2 \%$ de los adultos entre las edades de 21 y 49 años, y a menos del $2 \%$ de los adultos mayores de 50 años. De acuerdo con la Fundación Americana de la Tartamudez las personas que tartamudean son conscientes de su tartamudez y suelen dejar que dicha condición determine la vocación que eligen.

\section{Tartamudez y Trabajo}

La persona con tartamudez pudiera bloquearse, cometer repeticiones de sonidos, sílabas o palabras, hacer pausas inadecuadas que interfieran con la claridad del mensaje, prolongar sonidos o sílabas, o añadir expresiones como muletillas, que pudieran interferir en que el mensaje llegue con claridad. Algunas personas pudieran presentar movimientos asociados a la falta de fluidez, como, por ejemplo, fruncir el ceño, parpadear de manera continua, tensar alguna parte de su cuerpo u otros movimientos estereotipados que distraerán aún más al oyente (ASHA, 2021).

La reacción de los oyentes ante este cuadro es de asombro, enojo, malestar, pena, burla y, en muchas ocasiones, discrimen. Existe literatura sobre las implicaciones de la tartamudez en los niños, específicamente el acoso o bullying al cual son sometidos (Hernández, 2014). Según Langevin et al. (2009), el 81\% de los niños que tartamudea informó haber sufrido de bullying en algún momento de sus vidas, y el 56\% de estos indicó que fue acosado por su condición de tartamudez al menos una vez en semana. La literatura evidencia que la población que rodea a la persona con tartamudez, entiéndase: familiares, amistades, empleadores y maestros son los que manifiestan actitudes despectivas hacia las personas con esta condición (Castejón et al., 2005).

A pesar de lo expuesto referente a las reacciones de las personas que se relacionan con personas tartamudas, encontramos una encuesta que realizaron Iimura y Miyamoto (2021) que presenta unos datos muy interesantes. En la encuesta participaron trabajadores, personas que conocían a alguna persona que tartamudeaba en su trabajo, entre sus amistades o familiares y personas que estaban en posición de contratar individuos para ocupar puestos de trabajo. Iimura y Miyamoto encontraron que los participantes encuestados percibían que emplear a personas con tartamudez no tenía implicaciones negativas. En esta encuesta se reflejó que el conocer previamente a una persona con tartamudez en el ambiente laboral, mejoraba significativamente las actitudes positivas hacia este grupo. Concluyen los autores que el factor principal asociado a poseer estas actitudes positivas es la experiencia de tener contacto con personas que tartamudean en sus ambientes de trabajo. Este estudio destaca la importancia de conocer a este grupo y sus características para de esa forma poder tener una actitud más positiva hacia ellos. Aunque estos datos son muy alentadores, las experiencias laborales de las personas con tartamudez reflejan elementos diferentes, los cuales expondremos a continuación.

La falta de fluidez es un aspecto que se puede distinguir y percibir tanto en forma visual como auditivamente, no solo para quien la padece sino también para los que reciben el mensaje (Bloodstein et al., citados por Castejón et al., 2005). Por lo general, la percepción que tiene la mayor parte de los oyentes en relación con las personas con tartamudez es una despectiva, negativa y de discrimen. Numerosas investigaciones indican que las actitudes hacia las personas con tartamudez reflejan prejuicio, estereotipos negativos y estigma (Hughes et al., 2010; Carlo \& St. Louis, 2014).

En muchas ocasiones las limitaciones, prejuicios y actitudes negativas hacia la tartamudez pudieran surgir de los sentimientos de la misma persona que presenta esta condición. Esta actitud de minusvalía, inferioridad o limitación de capacidades pudiera originarse desde la niñez temprana a consecuencia del bullying que experimentaron. Esta conducta pudiera hacer que el niño con esta condición continúe durante su adolescencia y adultez, aumentando estos sentimientos de poca estima, llevándolo a tener problemas cuando se integre al campo laboral. Hernández (2014) hace énfasis en que la tartamudez es una de las condiciones dentro de los trastornos comunicológicos en las cuales se observa con mayor regularidad el bullying.

Guitar (1998), indica que las personas con tartamudez, especialmente los adultos, se sienten avergonzados de su condición. Debido a la repercusión que tiene la tartamudez en la comunicación, se puede observar un efecto negativo en las relaciones interpersonales y en la calidad de vida de las personas con la condición (Manning, 2001). Palasik et al. (2012), 
encontraron que los participantes de su investigación indicaron que su tartamudez afectaba algunos aspectos de sus experiencias ocupacionales y que sus empleadores habían hecho juicios negativos de ellos como resultado de su tartamudez durante entrevistas de empleo o al ser considerados para oportunidades de ascensos y progreso dentro de la compañía.

Klein y Hood (2004), encontraron que las personas que tartamudean creen que su condición interfiere con su desempeño laboral y dejan pasar ofertas de empleo o ascensos en sus empleos a consecuencia de su tartamudez. Según los autores, las personas que tartamudean pudieran estar mal remuneradas en sus empleos, no solo por las actitudes de la sociedad en relación con la tartamudez, sino también por sus propias actitudes. Estas actitudes pudieran entonces influir en sus posibilidades de empleo y progreso. Se esperaría entonces que la persona con tartamudez busque ayuda profesional e intervención clínica para mejorar su calidad de vida y para aumentar las posibilidades de mejores oportunidades de trabajo (Craig et al., 2009).

Rice y Kroll $(1997,2006)$ concluyeron en sus estudios que las personas con tartamudez creen que su condición repercute de manera negativa en su empleabilidad, su desempeño en el trabajo y en el progreso de su carrera. Interesantemente los autores indican que muchas veces los desafíos a los cuales se enfrentan las personas con tartamudez son causados por sus propios comportamientos. Los datos indican que las personas con tartamudez buscan empleos que requieran hablar poco y algunos no aceptan un trabajo nuevo o un ascenso debido a su condición.

Autores como Zhang y Kalinowski (2012), indican que entre los obstáculos que confrontan las personas que tartamudean, el más devastador, según su opinión, es la discriminación en el empleo y en las oportunidades educativas. Indican estos autores que las personas que tartamudean están expuestas a estereotipos negativos que les impiden obtener oportunidades de empleo y promociones. Para ellos, los estereotipos más comunes giran en torno a la impresión ampliamente aceptada de que los tartamudos son nerviosos, tímidos, callados, acomplejados, retraídos, tensos, ansiosos, miedosos, reticentes, y precavidos. Por otro lado, Rice y Kroll $(1997,2006)$ establecen que las personas con tartamudez encuentran retos en sus trabajos siendo el principal el confrontar problemas para ser promovidos a otros puestos, principalmente por su condición.

En la misma línea de lo que se expuso anteriormente, Silverman y Bongey (1997) encontraron que al comparar a las personas que tartamudean con las que no tartamudean, las personas con tartamudez son percibidas de forma menos positiva al realizar la misma tarea. Por ejemplo, existe la creencia de que en aquellos empleos donde se requieran competencias o destrezas verbales, tales como atender público en un área de recepción de una organización o contestar llamadas telefónicas, las personas con tartamudez tendrían serias dificultades.

De igual manera, Geerlach et al. (2018) realizaron un estudio donde compararon a personas con y sin tartamudez, tomando en consideración sus ingresos económicos y su género sexual. Los autores evidenciaron que la tartamudez se relacionaba, no solo a una reducción en ingresos por su condición de habla, sino también se observó discriminación por género, siendo las mujeres tartamudas las más afectadas.

El discrimen contra las personas con tartamudez en el área laboral se ve en cualquier parte del mundo. En países como los Estados Unidos, el enfoque en la diversidad en los contextos laborales se inició con esfuerzos concertados para proveer justicia social, protección de los derechos civiles y proteger a las personas afectadas por la exclusión y la discriminación (Ferdman, 2014). Weiner y Tetnowski (2016), señalan como ejemplo el American with Disabilities Act (ADA) según enmiendas del 2008, que provee mayores protecciones hacia ciertos grupos como son las personas con tartamudez. Se establece en la misma que es contra la ley discriminar contra personas con alguna discapacidad en áreas como empleo, recreación, institucionalización, servicios de salud, voto y acceso a servicios públicos.

Según Weiner y Tetnowski (2016), la ley ADA define persona con impedimento como una persona que tenga un impedimento físico o mental que limite en forma sustancial uno o más de las actividades principales de vida, que posea expediente de ese impedimento y que se considere que tiene dicho impedimento. Tomando en consideración esta definición de ADA, se sugiere entonces, que la tartamudez es un impedimento ya que limita la habilidad de una 
persona para hablar, comunicarse y trabajar. Weiner y Tetnowski indican, sin embargo, que para que se establezca la protección bajo la ley ADA, el individuo tendría que demostrar que la tartamudez representa un impedimento sustancial. Reconocer la tartamudez como un impedimento, ayudaría a que las personas con esta condición pudieran tener mayor protección y experimentar menos discrimen. A tono con este planteamiento, Venkatagiri (2009) expresa que al preguntarles a las personas con tartamudez si prefieren libertad o fluidez, en su mayoría estas indicaron que prefieren ser fluentes, por las consecuencias que la fluidez tiene en su vida personal y laboral.

Como se observa en los estudios que presentamos anteriormente, las experiencias laborales de las personas con tartamudez pueden verse matizadas por actitudes negativas y discriminatorias por parte de sus patronos y compañeros de trabajo. De igual forma, las personas con tartamudez entienden que sus oportunidades en el trabajo pueden verse afectadas por su condición. En Puerto Rico, no existe evidencia que documente la experiencia de trabajo de personas con tartamudez. Ante esta necesidad, la siguiente investigación tiene como propósito general obtener información sobre cómo es la experiencia de trabajo de personas con tartamudez. Específicamente deseamos auscultar: (1) ¿Qué dificultades encuentran en el contexto de trabajo? (2) ¿Cómo se relacionan con sus compañeros de trabajo? (3) ¿Cómo se perciben como trabajadores? (4) ¿Cómo es su relación con su tartamudez?

\section{MÉTODO}

\section{Diseño de Investigación}

Esta investigación es una de tipo cuantitativa, exploratoria y con un diseño no experimental con el propósito de comenzar a documentar la experiencia laboral de adultos con tartamudez en Puerto Rico. En este tipo de investigación no existe ningún tipo de manipulación de las variables ni asignación aleatoria de los participantes y nos permitirá conocer más en detalle un fenómeno poco estudiado en un contexto particular (Hernández Sampieri et al., 2010; Muchinsky \& Howes, 2019; Naupas Paitán, et al., 2018).

\section{Participantes}

Los participantes del estudio son 13 personas con tartamudez que trabajan, con 21 años o más de edad, que residen en Puerto Rico y que fueron contactados por disponibilidad. La mayoría de estos participantes son hombres $(62 \%)$ y sus edades fluctuaron entre los 21 y 60 años, siendo la edad promedio 31 años $(\mathrm{DE}=$ 10.24). Un $46 \%$ de los participantes indicó que poseía una preparación académica de bachillerato. El 77\% señaló que recibió o estaba recibiendo servicios de terapia del habla por su tartamudez al momento de la investigación. En términos laborales, un $58 \%$ indicó que trabajaba en el sector gubernamental. Referente al área de trabajo, un 50\% indicó laborar en ventas y en el área de la salud (25\% cada uno). Al auscultar el número de años que llevaban trabajando, observamos que los mismos fluctuaron entre los 2 y 30 años, con un promedio de 10 años de experiencia laboral $(\mathrm{DE}=7.70)$. Por otro lado, un $85 \%$ indicó que trabaja a tiempo completo y $75 \%$ señaló que no realiza tareas de supervisión.

\section{Instrumentos}

Cuestionario de datos generales. En este cuestionario se solicitó información referente al género sexual, edad, preparación académica, número de años trabajando, tipo de organización donde trabaja, tipo de industria para la cual trabaja, si realiza tareas de supervisión y tipo de contrato que tiene con la organización.

Escala de experiencia de tartamudez en el trabajo. Esta escala la desarrollaron los autores principales del estudio y tiene 36 aseveraciones, las cuales se responden utilizando una escala que fluctúa entre 1 (fuertemente en desacuerdo) a 6 (fuertemente de acuerdo). En estas aseveraciones se ausculta las experiencias que ha tenido en su lugar de trabajo, relación con sus compañeros de trabajo, relación entre su tartamudez y sus tareas de trabajo y su relación con la tartamudez. Un ejemplo de un ítem de esta escala es: "He sido despedido de mi trabajo". Además, la escala tiene una lista de 12 características para que el/la participante indique cuáles lo describen como persona (por ejemplo: Dinámico/a). El índice de confiabilidad alfa de Cronbach para la escala es de .80 .

\section{Procedimientos Generales}

Este estudio contó con la aprobación del IRB de la Universidad Albizu Recinto de San Juan para su realización (SP 19-29). Se contactó una muestra por conveniencia de 13 personas con tartamudez a quienes se 
les explicó el propósito del estudio y se le informó sobre sus derechos como participante. Una vez la persona accedió a participar en el estudio tuvo acceso a los instrumentos para recopilar los datos. Al finalizar la recopilación de los datos se procedió a realizar los análisis descriptivos correspondientes utilizando el programa estadístico IBM SPSS. Para la descripción de la muestra se realizaron análisis de promedios y desviación estándar para las variables de edad y años de servicio; para las otras variables demográficas se analizó el porcentaje de respuesta a las alternativas provistas en cada variable. Por otro lado, para conocer las respuestas de los participantes a la Escala de experiencia de tartamudez en el trabajo se analizó el porcentaje que seleccionó cada una de las alternativas de respuesta de los reactivos que se incluyeron en la misma. Todos los datos obtenidos de los participantes se utilizaron para los análisis estadísticos que se realizaron; no hubo la necesidad de eliminar ninguno por estar incompleta la información.

\section{RESULTADOS}

A continuación, presentaremos los principales resultados que encontramos al administrar el cuestionario a personas con tartamudez. Los resultados los presentaremos tomando como base las siguientes categorías: dificultades en su trabajo, relaciones con supervisores y compañeros de trabajo, relación con su trabajo, relación con su tartamudez y características que los describen como personas. En el texto presentamos un porcentaje que representa la suma de los tres niveles de la categoría de respuesta correspondiente a de acuerdo (levemente de acuerdo, moderadamente de acuerdo y fuertemente de acuerdo). Le daremos atención a los porcentajes generales más altos. En las tablas presentamos las respuestas a todos los reactivos que se incluyeron en el instrumento tomando en consideración los tres niveles de la categoría de acuerdo según aparecían en dicho instrumento.

Iniciaremos presentando los principales problemas o dificultades que identificaron los participantes en el trabajo. Como se puede observar en la Tabla 1 estos problemas giraron principalmente en torno a no ser considerados para proyectos importantes $(77 \%)$, no ser seleccionados para un puesto de trabajo a pesar de sus credenciales profesionales $(69 \%)$, ser evaluados injustamente $(62 \%)$ y perder oportunidades de trabajo (54\%).
$\mathrm{Al}$ auscultar cómo los participantes describen su relación con los compañeros de trabajo y los supervisores encontramos datos muy interesantes. Desde el punto de vista positivo podemos observar en la Tabla 2 que los participantes nos indicaron que sus compañeros de trabajo le dan mucho apoyo (54\%), los tratan como iguales (54\%) y no perciben un trato desigual por su condición (46\%). Por otro lado, los participantes indicaron ser objeto de chistes (77\%) y burlas (54\%) por su manera de hablar, el/la supervisor/a evita que hagan presentaciones orales (46\%), perciben que sus compañeros le tienen pena (46\%) y que los/as compañeros/as delegan en otra persona y no en ellos/as el presentar asuntos importantes en reuniones de trabajo (38\%).

La tercera dimensión que se auscultó fue la relación del participante con su trabajo. Como observamos en la Tabla 3 los participantes de la investigación nos indicaron que les da miedo dirigir reuniones de grupo $(77 \%)$, les incomoda hablar en reuniones de trabajo (69\%), evitan hacer presentaciones orales $(61 \%)$, y no se atreven a asistir a adiestramientos fuera del lugar de trabajo (61\%). Por otro lado, también indicaron que se sienten seguros de sus capacidades para realizar su trabajo $(61 \%)$, se sienten muy satisfechos con el trabajo que tienen (54\%) y entienden que la tartamudez no les limita en su trabajo (31\%).

Tabla 1

Dificultades encontradas en su trabajo por su tartamudez.

\begin{tabular}{|c|c|c|c|}
\hline & $\begin{array}{l}\text { Levemente } \\
\text { de acuerdo }\end{array}$ & $\begin{array}{c}\text { Moderadamente } \\
\text { de acuerdo }\end{array}$ & $\begin{array}{c}\text { Fuertemente } \\
\text { de acuerdo }\end{array}$ \\
\hline $\begin{array}{l}\text { Perdió oportunidades } \\
\text { de trabajo. }\end{array}$ & 15.4 & 30.8 & 7.7 \\
\hline $\begin{array}{l}\text { No fue considerado } \\
\text { para proyectos im- } \\
\text { portantes. }\end{array}$ & 23.1 & 46.2 & 7.7 \\
\hline $\begin{array}{l}\text { Le negaron la oportu- } \\
\text { nidad de ocupar } \\
\text { puestos de mayor je- } \\
\text { rarquía. }\end{array}$ & 8.3 & 16.7 & 25.0 \\
\hline $\begin{array}{l}\text { Fue evaluado injusta- } \\
\text { mente. }\end{array}$ & 30.8 & 23.1 & 7.7 \\
\hline Fue despedido. & - & 8.3 & - \\
\hline $\begin{array}{l}\text { No fue seleccionado } \\
\text { para un puesto de tra- } \\
\text { bajo a pesar de sus } \\
\text { credenciales profesio- } \\
\text { nales. }\end{array}$ & 38.5 & 30.8 & - \\
\hline
\end{tabular}


Tabla 2

Relaciones con supervisores y compañeros de trabajo

\begin{tabular}{lccc}
\hline & $\begin{array}{c}\text { Levemente } \\
\text { de acuerdo }\end{array}$ & $\begin{array}{c}\text { Moderada- } \\
\text { mente de } \\
\text { acuerdo }\end{array}$ & $\begin{array}{c}\text { Fuertemente } \\
\text { de acuerdo }\end{array}$ \\
\hline $\begin{array}{l}\text { Compañeros de trabajo evi- } \\
\text { tan acercarse. }\end{array}$ & 15.4 & - & - \\
$\begin{array}{l}\text { Supervisor evita que haga } \\
\text { presentaciones orales. }\end{array}$ & 23.1 & 15.4 & 7.7 \\
$\begin{array}{l}\text { Compañeros delegan en otra } \\
\text { persona el presentar asuntos } \\
\text { importantes en reuniones de } \\
\text { trabajo. }\end{array}$ & 30.8 & 7.7 & - \\
$\begin{array}{l}\text { Es objeto de chistes. } \\
\begin{array}{l}\text { La gente no le tiene pacien- } \\
\text { cia. }\end{array}\end{array}$ & 23.1 & 7.7 & - \\
$\begin{array}{l}\text { Percibe que compañeros le } \\
\text { tienen pena. }\end{array}$ & 23.1 & 15.4 & 7.7 \\
$\begin{array}{l}\text { La gente se burla de su ma- } \\
\text { nera de hablar. }\end{array}$ & 7.7 & 30.8 & 15.4 \\
$\begin{array}{l}\text { Compañeros le dan mucho } \\
\text { apoyo. }\end{array}$ & 23.1 & 15.4 & 15.4 \\
$\begin{array}{l}\text { Compañeros lo tratan como } \\
\text { otro igual. }\end{array}$ & 23.1 & 15.4 & 15.4 \\
$\begin{array}{l}\text { No percibe trato desigual } \\
\text { por su condición. }\end{array}$ & 30.8 & 7.7 & 7.7 \\
\hline
\end{tabular}

Tabla 3

Relación con su trabajo.

\begin{tabular}{lccc}
\hline & $\begin{array}{c}\text { Levemente } \\
\text { de acuerdo }\end{array}$ & $\begin{array}{c}\text { Moderada- } \\
\text { mente de } \\
\text { acuerdo }\end{array}$ & $\begin{array}{c}\text { Fuertemente } \\
\text { de acuerdo }\end{array}$ \\
\hline $\begin{array}{l}\text { Evita hacer presentaciones } \\
\text { orales. }\end{array}$ & 23.1 & 23.1 & 15.4 \\
$\begin{array}{l}\text { Le incomoda hablar en } \\
\text { reuniones de trabajo. }\end{array}$ & 30.8 & 15.4 & 23.1 \\
$\begin{array}{l}\text { Le da miedo dirigir reunio- } \\
\text { nes de grupo. }\end{array}$ & 15.4 & 38.5 & 23.1 \\
$\begin{array}{l}\text { No se atreve asistir a adies- } \\
\text { tramientos fuera del lugar } \\
\text { de trabajo. }\end{array}$ & 38.5 & 23.1 & - \\
$\begin{array}{l}\text { Se siente muy satisfecho con } \\
\text { el trabajo que tiene. }\end{array}$ & - & 15.4 & 23.1 \\
$\begin{array}{l}\text { La tartamudez no le limita } \\
\text { en su trabajo. }\end{array}$ & 23.1 & 7.7 & - \\
$\begin{array}{l}\text { Se siente seguro de sus capa- } \\
\text { cidades en el trabajo. }\end{array}$ & 15.4 & 30.8 & 15.4 \\
$\begin{array}{l}\text { Le da ansiedad si tiene que } \\
\text { expresarse en reuniones. }\end{array}$ & 7.7 & 38.5 & 7.7 \\
$\begin{array}{l}\text { Prefiere no hablar en reunio- } \\
\text { nes de trabajo. }\end{array}$ & 15.4 & 38.5 & - \\
\hline
\end{tabular}

En la cuarta dimensión, auscultamos la relación de los participantes con la tartamudez. Los participantes nos indicaron, entre otras cosas, que entienden que tendrían un mejor trabajo, pero su tartamudez se lo impidió (62\%), experimentan mucho coraje al no poder comunicarse efectivamente (54\%), se sienten frustrados al no poderse comunicar efectivamente (53\%), no solicitan trabajos que conlleven hablar en público (54\%) y se deprimen cuando no pueden comunicarse efectivamente $(53 \%)$. Sin embargo, un $77 \%$ indicó que, a pesar de su tartamudez, se comunica efectivamente (ver Tabla 4).

Por último, le pedimos a los participantes que marcaran las características que lo describen como persona. Aquí observamos que la mayoría se describe con buen sentido del humor (69\%), competente $(62 \%)$, simpático $(62 \%)$, inteligente y sociable $(54 \%$ cada uno). No obstante, un $46 \%$ se describió como inseguro, callado y como una persona que evita situaciones donde tenga que hablar (ver Tabla 5).

\section{DISCUSIÓN}

La presente investigación tuvo como propósito principal documentar la experiencia de trabajo de un grupo de personas con tartamudez en el contexto laboral en Puerto Rico. Como observamos en los resultados que se presentaron anteriormente, esta experiencia está matizada por elementos tanto positivos como negativos de acuerdo con la información que proveyeron los participantes. Por un lado, observamos que los participantes nos indican que sus compañeros de trabajo los aceptan y los tratan como iguales, pero, por otro lado, también nos indican que son objeto de bromas de parte de ellos. De igual forma vemos que los participantes nos indican que han presentado problemas en sus trabajos experimentando hasta cierto punto discrimen por su condición de tartamudez. Estos resultados son cónsonos con los datos obtenidos en otras investigaciones (Hughes et al., 2010; Klein \& Hood, 2004; Palasik et al., 2012; Rice \& Kroll, 1997, 2006; Zhang \& Kalinowski, 2012) donde se evidencia esta experiencia de trato desigual y discrimen por su tartamudez.

Estos resultados nos plantean la necesidad de tener contextos de trabajo donde haya más inclusión y trato de respeto para todos sus integrantes partiendo de sus elementos de diversidad. Si presumimos que la fuerza laboral en todos los contextos es una más 
diversa, entonces se necesitan desarrollar políticas y prácticas organizacionales que promuevan la inclusión (DeNisi, 2014). Estas iniciativas deben diseñarse con el propósito de que tanto los individuos, las organizaciones y la sociedad se beneficien de la diversidad (DeNisi, 2014). En este esfuerzo según DeNisi, los líderes deben ser un ejemplo y las personas responsables para establecer el clima donde se valore la inclusión.

Un elemento que debemos resaltar de nuestros resultados es la auto percepción de nuestros participantes. Por un lado, indican que son muy capaces para realizar las tareas que se le asignan en sus trabajos, pero, por otro lado, señalan que su tartamudez representa un impedimento en el contexto laboral y se limitan o cohiben en el desempeño de ciertas tareas. Como se observó previamente, en otras investigaciones se encontraron resultados similares tendientes a la propia percepción que tienen las personas con tartamudez y cómo eso les limita en sus trabajos (Guitar, 1998; Klein \& Hood, 2004; Rice \& Kroll, 1997, 2006). Ferdman y Morgan (2014) resaltan que, en la práctica de la inclusión, lo primero que debe darse es la aceptación del individuo mismo como persona, con sus fortalezas y desafíos. La clave para aceptarse debe basarse en quién fue uno en el pasado y en sus aspiraciones y deseos, en lugar de tratar de ser alguien diferente (Ferdman \& Morgan, 2014). Recalcan estos autores que los líderes y las organizaciones deben crear las condiciones para que las personas también se incluyan a sí mismos completamente como resultado de experimentar un ambiente de aceptación de la persona tal cual es.

La inclusión tiene que ver con cómo las organizaciones, sus grupos, sus líderes y sus miembros proveen las formas que permitan que todo el mundo, tomando en consideración todos los tipos de diferencias, puedan participar, contribuir, tener una voz, sentirse conectados y pertenecer sin perder su unicidad ni perder elementos de valor de su identidad (Ferdman, 2014). Es por eso por lo que debemos combatir el sentimiento de exclusión que informaron varios de nuestros participantes en el estudio.
Tabla 4

Dificultades encontradas en su trabajo por su tartamudez.

\begin{tabular}{|c|c|c|c|}
\hline & $\begin{array}{l}\text { Levemente } \\
\text { de acuerdo }\end{array}$ & $\begin{array}{c}\text { Moderadamente } \\
\text { de acuerdo }\end{array}$ & $\begin{array}{c}\text { Fuertemente } \\
\text { de acuerdo }\end{array}$ \\
\hline $\begin{array}{l}\text { Se conforma con el trabajo } \\
\text { que tiene y no aspira a uno } \\
\text { de mayor responsabilidad. }\end{array}$ & 23.1 & 7.7 & 7.7 \\
\hline $\begin{array}{l}\text { Escogió esta carrera cuando } \\
\text { la que le interesaba era otra. }\end{array}$ & 7.7 & - & 30.8 \\
\hline $\begin{array}{l}\text { Se siente frustrado al no po- } \\
\text { der comunicarse efectiva- } \\
\text { mente. }\end{array}$ & 23.1 & 15.4 & 14.4 \\
\hline $\begin{array}{l}\text { Se deprime cuando no } \\
\text { puede comunicarme efecti- } \\
\text { vamente. }\end{array}$ & 23.1 & 15.4 & 15.4 \\
\hline $\begin{array}{l}\text { Experimenta mucho coraje } \\
\text { al no poder comunicarse } \\
\text { efectivamente. }\end{array}$ & 23.1 & 23.1 & 7.7 \\
\hline $\begin{array}{l}\text { Tendría un mejor trabajo si } \\
\text { no fuera porque es tarta- } \\
\text { mudo. }\end{array}$ & 30.8 & - & 30.8 \\
\hline $\begin{array}{l}\text { Se siente muy orgulloso de } \\
\text { ser tartamudo. }\end{array}$ & 23.1 & - & 7.7 \\
\hline $\begin{array}{l}\text { No le preocupa para nada su } \\
\text { tartamudez. }\end{array}$ & 15.4 & - & - \\
\hline $\begin{array}{l}\text { A pesar de su tartamudez, se } \\
\text { comunica efectivamente. }\end{array}$ & 38.5 & 23.1 & 15.4 \\
\hline $\begin{array}{l}\text { La tartamudez no ha afec- } \\
\text { tado sus oportunidades de } \\
\text { empleo. }\end{array}$ & 15.4 & - & 7.7 \\
\hline $\begin{array}{l}\text { No solicita trabajos que con- } \\
\text { lleven hablar en público. }\end{array}$ & 46.2 & 7.7 & - \\
\hline
\end{tabular}

Tabla 5

Características que lo describen como persona

\begin{tabular}{|c|c|c|c|c|c|}
\hline Característica & $n$ & $\%$ & Característica & $n$ & $\%$ \\
\hline Inteligente & 7 & 54 & Callado/a & 6 & 46 \\
\hline Nervioso/a & 1 & 8 & Activo/a & 4 & 31 \\
\hline Simpático/a & 8 & 62 & $\begin{array}{l}\text { Evita situacio- } \\
\text { nes donde } \\
\text { tenga que ha- } \\
\text { blar }\end{array}$ & 6 & 46 \\
\hline Pasivo/a & 4 & 31 & Sociable & 7 & 54 \\
\hline Inseguro/a & 6 & 46 & $\begin{array}{l}\text { Con buen sen- } \\
\text { tido del humor }\end{array}$ & 9 & 69 \\
\hline Dinámico/a & 5 & 39 & Competente & 8 & 62 \\
\hline
\end{tabular}

Nota: Otras características con 7\% cada una: distraído, empático, frustrado, deprimido 
Por otro lado, los resultados de este estudio señalan la importancia, no solo de la aceptación, inclusión y empatía hacia las personas con tartamudez, sino también de la importancia de educar hacia la diversidad. Ese sentimiento de exclusión que indicaron los participantes en sus respuestas pudiera ser minimizado si las personas estuvieran orientadas sobre lo que es la tartamudez y sus implicaciones en el área emocional, académica y laboral. Decir que los compañeros de trabajo los aceptan, pero a su vez ser motivo de burlas y chistes evidencia, no solo una falta de sensibilidad, sino también una falta de conocimiento sobre lo que implica tener tartamudez.

Como toda investigación, nuestro estudio presenta como principal desafío el número reducido de participantes con el que contó, lo cual sabemos, afecta la generalización de sus resultados. A pesar de ello, nuestros datos presentan un cuadro de la realidad que experimenta un grupo de personas con tartamudez que trabajan en varias organizaciones en Puerto Rico. Confiamos que estos resultados motiven a otros investigadores a profundizar en el tema para de esa forma darle voz a este grupo y poder generar ambientes de trabajo donde experimenten bienestar y se sientan valorados e incluidos. Nuestro estudio, es el primer esfuerzo por darle esa voz a las personas integrantes de este grupo para expresar sus vivencias en los contextos de trabajo en Puerto Rico. Esperamos haber creado conciencia de la importancia de la diversidad funcional en los ambientes laborales y del reclamo por una práctica de la inclusión que sea de beneficio para el individuo, la organización y la sociedad.

\section{CONCLUSIÓN}

La experiencia de trabajar en una organización puede representar retos y desafíos pues las interacciones entre las personas que componen la fuerza laboral es terreno fértil para ello. Sin embargo, los resultados de nuestro estudio nos muestran que, si a esos retos se le añade el vivir con tartamudez, esa experiencia podría estar matizada por actitudes negativas y discriminatorias hacia ese grupo en particular. Muchas veces la falta de información sobre la condición o la falta de experiencia con personas que la posean puede llevar al desarrollo de esas actitudes.

Es por esto por lo que las organizaciones deben tener entre sus valores la inclusión y el respeto a la diversidad. Particularmente, se debe tener una visión más abarcadora de lo que representa la diversidad (más allá de lo que es el género, la edad, la raza o la orientación sexual, por mencionar algunas) y entender que la diversidad funcional, particularmente en el área de la comunicación, también debe formar parte de los programas organizacionales que buscan el manejo adecuado de las diferencias entre las personas en los contextos laborales. Solo así podremos decir que se valora la diversidad y nos enriquecemos como organización de las diferencias entre los seres humanos.

Financiamiento: La presente investigación no fue financiada por alguna entidad ni patrocinador.

Conflicto de Intereses: No existen conflictos de intereses de parte de los autores de la investigación.

Aprobación de la Junta Institucional para la Protección de Seres Humano en la Investigación: IRB de la Universidad Albizu Recinto de San Juan (SP 19-29).

Consentimiento o Asentimiento Informado: Todo participante completó un consentimiento informado.

Proceso de Revisión: Este estudio ha sido revisado por pares externos en modalidad de doble ciego.

\section{REFERENCIAS}

ASHA. (2021). ¿Qué es la tartamudez? https://www.asha.org/public/speech/spanish/la-tartamudez/

Carlo, E., \& St. Louis, K. (2014). Actitudes de adultos puertorriqueños hacia la tartamudez y hacia el tartajeo. Revista OPPHLA, 32(1), 16-21.

Castejón, L., Núñez, J., \& González Pienda, J. (2005). Contenido del estereotipo de los maestros sobre los alumnos tartamudos. Psicothema. 17(3). 496-501.

Craig, A.R., Blumgart, E. \& Tran, Y. (2009). The impact of stuttering on the quality of life in adult people who stutter. Journal of Fluency Disorders, 34, 61-71. https://doi.org/10.1016/j.jfludis.2009.05.002

De Anca, C. \& Vázquez, A. (2007). Managing diversity in the global organization. Palgrave Macmillan. https://doi.org/10.1057/9780230800885

DeNisi, A.S. (2014) An I/O Psychologist's perspective on diversity and inclusion in the workplace. En B.M. Ferdman \& B.R. Deane (Eds.), Diversity at work: The practice of inclusion (pags. 564-579). John Wiley \& Sons, Inc.

Ferdman, B.M. (2014). The practice of inclusion in diverse organizations: Toward a systemic and inclusive framework. En B.M. Ferdman \& B.R. Deane (Eds.), Diversity at work: The practice of inclusion (pags. 3-54). John Wiley \& Sons, Inc.

Ferdman, B.M. \& Morgan, L. (2014). Creating inclusion for oneself: 
Knowing, accepting, and expressing one's whole self at work. En B.M. Ferdman \& B.R. Deane (Eds.), Diversity at work: The practice of inclusion (pags. 93-127). John Wiley \& Sons, Inc.

Fundación Americana de la Tartamudez (2021). ¿Sabías que...? (Did you know...). https://www.tartamudez.org/sabias-quedid-you-know

Geerlach, H.,Totty, E., Subramanian, A., \& Zebrowsky, P. (2018). Stuttering and labor market in the United States. Journal of Speech, Language, and Hearing Research, 61(7), 1649-1663. https://doi.org/10.1044/2018_JSLHR-S-17-0353

Guitar, B. (1998). Stuttering: an integrated approach to its nature and treatment. Williams and Wilkins.

Hernández, M. (2014). Bullying y tartamudez: Una breve revisión de literatura. Ciencias de la Conducta. 29 (1), 9-19.

Hernández Sampieri, R., Fernández Collado, C. \& Baptista Lucio, M.P. (2010). Metodología de investigación. McGraw Hill.

Hughes, S., Gabel, R., Irani, F. \& Schlagheck, A. (2010). University student's explanations for their description of people who stutter. An exploratory mixed model story. Journal of Fluency Disorders, 35, 280-298. https://doi.org/10.1016/j.jfludis.2010.05.006

Iimura, D., \& Miyamoto, S. (2021). Public attitudes toward people who stutter in the workplace: A questionnaire survey of Japanese employees. Journal of Communication Disorders, 89. https://doi.org/10.1016/j.jcomdis.2020.106072

Jayne, M. \& Dipboye, R. L. (2004). Leveraging diversity to improve business performance: Research findings and recommendations for organizations. Human Resource Management, 43(4), 409-424. https://doi.org/10.1002/hrm.20033

Klein, J.F., \& Hood, S.B. (2004). The impact on stuttering on employment opportunities and job performance. Journal of Fluency Disorders, 29, 255-273. https://doi.org/10.1016/j.jfludis.2004.08.001

Langevin, M., Packman, A. \& Onslow, M. (2009). Peer responses to stuttering in the preschool setting. American Journal of SpeechLanguage Pathology, 18, 264-276. http://dx.doi.org/10.1044/10580360(2009/07-0087

Manning, W.H. (2009). Clinical decision making in fluency disorders (3rd ed.). Singular-Thomson.

Mendyra.com. (2021). ¿Qué es la tartamudez? https://www.mindyra.com/es-cr/solutions/adults/stuttering

Muchinsky, P. \& Howes, S.S. (2019). Psychology applied to work. Hypergraphic Press.

Ñaupas Paitán, H., Valdivia Dueñas, M.R., Palacios Vilela, J.J. \& Romero Delgado, H.E. (2018). Metodología de la investigación cuantitativa-cualitativa y redacción de la tesis. Ediciones de la U.

Palasik, S., Gabel, R., Hughes, C., \& Rusnak, E. (2012). Perceptions about occupational experiences by people who stutter. SGI 4 Perspectives on Fluency and Fluency Disorders, 22, 22-23. https://doi.org/10.1044/ffd22.1.22

Rice, M., \& Kroll, R. (1997). Workplace experiences of people who stutters. Journal of Fluency Disorders, 22, 140.

Rice, M., \& Kroll, R. (2006). The impact of stuttering at work: Challenges and discrimination. International Stuttering Awareness Day Online Conference http://www.mnsu.edu/comdis/isad9/isadcon9.html

Romero Flores, E. \& Villanueva Flores, M. (2016). La gestión de la diversidad en las organizaciones: Estudio de casos en el Campo de Gibraltar. Gestión Joven, 15, 26-35.

Silverman, F.H., \& Bongey, T.A. (1997). Nurses' attitudes toward physicians who stutter. Journal of Fluency Disorders, 22, 61-62.

Triandis, H. (2003). The future of workforce diversity in international organisations: A commentary. Applied Psychology: An International Review, 52(3), 486-496. https://doi.org/10.1111/14640597.00146

Venkatagiri, H.S. (2009). What do people who stutter want-fluency or freedom? Journal of Speech, Language and Hearing Research, 52, 500-515. https://doi.org/10.1044/1092-4388(2008/07-0019)

Weiner, C., \& Tetnowski, J. (2016). Stuttering discrimination under the law. Here's a look at how the law applies. The ASHA Leader, 21, 52-57. https://doi.org/10.1044/leader.FTR2.21072016.52

Zhang, J., \& Kalinowski, J. (2012). Culture and listeners gaze responses to stuttering. International Journal of Language \& Communication Disorders, 47(4), 388-397. https://doi.org/10.1111/j.14606984.2012.00152.x 